\title{
Meta
}

Journal des traducteurs

Translators' Journal

\section{Entre la transparence et l'opacité : traduire l'altérité québécoise}

\section{Patricia Godbout}

Volume 45, numéro 1, avril 2000

La traduction littéraire au Canada

Literary Translation in Canada

URI : https://id.erudit.org/iderudit/002927ar

DOI : https://doi.org/10.7202/002927ar

Aller au sommaire du numéro

Éditeur(s)

Les Presses de l'Université de Montréal

ISSN

0026-0452 (imprimé)

1492-1421 (numérique)

Découvrir la revue

Citer cet article

Godbout, P. (2000). Entre la transparence et l'opacité : traduire l'altérité québécoise. Meta, 45(1), 29-36. https://doi.org/10.7202/002927ar

\section{Résumé de l'article}

Examen du rôle particulier joué par les principaux traducteurs de littérature, du français vers l'anglais, à la fin des années 1950 et au début des années 1960. Analyse de leur" discours" sur la traduction, de leur rôle de médiation entre les "deux solitudes", du choix des livres à traduire, des stratégies de traduction. d'utilisation que vous pouvez consulter en ligne.

https://apropos.erudit.org/fr/usagers/politique-dutilisation/ 


\title{
Entre la transparence et l'opacité: traduire l'altérité québécoise
}

\author{
PATRICIA GODBOUT \\ Université de Sherbrooke
}

\begin{abstract}
RÉSUMÉ
Examen du rôle particulier joué par les principaux traducteurs de littérature, du français vers l'anglais, à la fin des années 1950 et au début des années 1960. Analyse de leur «discours » sur la traduction, de leur rôle de médiation entre les «deux solitudes», du choix des livres à traduire, des stratégies de traduction.
\end{abstract}

\begin{abstract}
An examination of the specific role played by major literary translators from French to English in the late 1950s and the early 1960s; an analysis of their "discourse" on translation, their role as mediator between the "two solitudes", their choice of works for translation and their translation strategies.
\end{abstract}

\section{MOTS-CLÉS/KEYWORDS}

traduction littéraire, Québec, altérité, rôle de médiation des traducteurs, stratégies de traduction

En décembre 1968, paraît dans Meta un texte intitulé «French Canadian Literature in Translation », par Philip Stratford ${ }^{1}$. Il s'agit du texte d'une conférence prononcée au premier congrès annuel du Conseil canadien des professeurs d'anglais, tenu à Calgary du 21 au 24 août 1968, dans laquelle Stratford fait le point sur l'état de la littérature canadienne-française en traduction anglaise.

La parution de cet article à la fin des années 1960 n'est pas fortuite. Elle signale un intérêt marqué, de la part d'une certaine élite intellectuelle canadienne-anglaise, à l'égard de la littérature québécoise, alors en plein essor. L'élément central de cette présentation est une bibliographie sélective d'ouvrages canadiens-français parus en traduction. Stratford devait poursuivre cette recherche, qui sera publiée en 1975 sous le titre de Bibliographie de livres canadiens traduits de l'anglais au français et du français à l'anglais, et rééditée en 1977 sous le même titre.

S'il trouve désolant le petit nombre de livres canadiens-français en traduction, l'auteur se console en constatant que le nombre de livres canadiens-anglais traduits en français est encore plus restreint: ainsi, il a recensé au total douze romans canadiens-anglais traduits en français, depuis le $\mathrm{XIX}^{\mathrm{e}}$ siècle! Stratford explique qu'il s'est rendu compte de cet état de choses au moment de préparer une série de cours sur les romanciers canadiens-anglais destinés à l'éducation des adultes à l'Université de Montréal. Les livres à l'étude devaient être disponibles en version française. Le choix s'est opéré de lui-même, dit-il, car il n'y avait que quatre romans traduits (à part la série Jalna de Mazo de la Roche, quelques ouvrages mineurs et les livres de Malcolm Lowry, dont l'appartenance aux lettres canadiennes est discutable): trois de Hugh MacLennan, Deux solitudes (1963) / Two Solitudes (1945), Le temps tournera au beau 
(1966) / Barometer Rising (1941) et Le matin d'une longue nuit (1967) / The Watch That Ends the Night (1959), et un de Mordecai Richler, L'apprentissage de Duddy Kravitz (1960) / The Apprenticeship of Duddy Kravitz (1959)2.

Stratford démolit l'excuse classique généralement servie pour expliquer le petit nombre de livres canadiens-anglais traduits en français: apparemment, les lecteurs francophones seraient bilingues et liraient ces œuvres directement en anglais. Mais selon Pierre Tisseyre, du Cercle du livre de France, seulement dix pour cent des lecteurs seraient dans cette situation. Et d'ailleurs, même les lecteurs bilingues ont une langue de lecture de prédilection.

Comme exemple de la situation moins désastreuse en traduction du français vers l'anglais, il donne les six titres sur 63 de la collection «New Canadian Library» de McClelland \& Stewart qui sont des traductions (de Gabrielle Roy, Ringuet et Roger Lemelin). Le Conseil des arts, dit-il, subventionne la majorité des traductions depuis 1959 (le programme d'aide à la traduction lui-même ne sera créé qu'en 1972). Stratford donne ensuite les mauvaises raisons souvent invoquées pour expliquer le si petit nombre de traductions littéraires au pays: faible intérêt de la part du public lecteur, réticence des éditeurs à investir dans la promotion à grande échelle de ces livres (publiés seulement pour faire preuve de «bonne volonté nationale»), manque de traducteurs compétents et d'incitatifs pour ceux qui le sont.

Dans la bibliographie sélective qu'il présente dans ce numéro de Meta, Stratford dresse une liste de 48 auteurs et 66 titres, ce qui n'est pas un si mauvais bilan... à première vue seulement, selon lui. En effet, nombre de ces livres sont épuisés; pour la poésie, on n'a bien souvent que des morceaux choisis; et la qualité laisse parfois beaucoup à désirer.

Une bonne partie du texte de Stratford est un plaidoyer en faveur de la traduction littéraire, présentée comme un outil de rapprochement entre les deux entités culturelles canadiennes:

La traduction est un pont entre les gens, et au Canada nous avons grandement besoin de plus de ponts et de ponts plus solides de ce genre. [...] Nos hommes politiques, économistes, historiens, commissaires des enquêtes royales sont traduits. Mais, à long terme, quand il s'agit de connaître un autre peuple, de savoir ce qu'il pense, sent, imagine, pour connaître ses réactions et ses souvenirs, il est beaucoup plus important de traduire nos poètes, dramaturges, romanciers, essayistes et journalistes ${ }^{3}$. (Stratford 1968: 183)

Stratford invite enfin les professeurs d'anglais à intégrer la littérature canadiennefrançaise en traduction à leurs programmes. Il y a longtemps, dit-il, que la littérature anglaise s'est approprié Don Quichotte, Crime et châtiment et La chartreuse de Parme et qu'elle a transformé en auteurs anglais Ibsen, Strindberg, Genet et Ionesco.

Le désir qui anime Stratford est celui de faire connaître aux lecteurs anglophones du Canada une littérature d'expression française issue de leur propre pays. Il la juge digne d'intérêt pour ses qualités intrinsèques, mais il est évident que le cadre dans lequel Stratford situe sa réflexion est canadien. C'est d'ailleurs précisément ce que lui reprochera, beaucoup plus tard, Chantal de Grandpré, dans un article intitulé «La canadianisation de la littérature québécoise: le cas Aquin», paru dans Liberté, en 1985. Cette dernière ne manquera pas de relever des mots comme «appropriation» et «assimilation» utilisés par l'auteur, pour prêter à celui-ci des visées annexionnistes.

L'article de $\mathrm{M}^{\mathrm{me}}$ de Grandpré nous permet de constater que les efforts de traduc- 
tion de la littérature canadienne-française, que d'aucuns perçoivent comme un signe d'ouverture à l'Autre et de respect de son altérité, prennent une tout autre signification lorsqu'ils sont placés dans la perspective du nationalisme étroit, où ils deviennent alors synonymes de conspiration. Qui dit traduction dit trahison, n'est-ce pas? Cela nous rappelle en outre que ni l'activité traduisante ni le discours qu'on tient sur celle-ci ne sont neutres ou anodins: ils participent à la redéfinition des paradigmes littéraires.

F. R. Scott, poète, traducteur et professeur de droit constitutionnel à l'Université McGill, fait paraître en 1962 St-Denys Garneau/Anne Hébert. Translations/Traductions, aux Éditions Klanak, à Vancouver. Dans une note du traducteur faisant suite à la préface de Gilles Marcotte, Scott donne un aperçu de sa poétique de la traduction. Concernant le choix des textes, il dit: "J'ai traduit ces poèmes parce que je les trouvais représentatifs des meilleures œuvres des deux poètes canadiens-français que j'admire le plus» (Scott 1962: 9) ${ }^{4}$. Il y a donc un choix critique qualitatif, de la part du traducteur, d'abord des poètes eux-mêmes, ensuite des poèmes à traduire. Selon Scott, meilleur est le poème original, meilleures sont les chances qu'une traduction, même imparfaite, soit valable.

Frank Scott expose ensuite sa méthode de traduction: "En traduisant, mon but principal est de modifier le poème le moins possible et de le laisser parler pour luimême dans une autre langue. Cela suppose d'opter pour la littéralité de préférence à des versions de remplacement, pour un seul poème en deux langues de préférence à deux poèmes similaires» (Scott 1962: 9). Dans une recension de ce recueil paru dans Canadian Literature, Léandre Bergeron va jusqu'à dire:

Sa traduction est absolument littérale. [.. .] Cette façon donne quelque fois du drôle d'anglais mais le respect du poème original qui anime le traducteur excuse facilement ces tournures, et même, on peut dire que cette traduction est d'autant plus valable qu'elle nous donne vraiment l'impression qu'un canadien-français est en train de nous parler dans sa langue seconde. (Bergeron 1963: 75)

Pour le poète et traducteur D. G. Jones, le désir de traduire la littérature québécoise qui a animé plusieurs écrivains et traducteurs anglophones à la fin des années 1960 correspond en fait à un changement d'attitude. Ainsi, à propos de la contribution de F. R. Scott comme traducteur, il écrit: "Le point de départ et d'arrivée de F. R. Scott, c'est le Québec. Vivre au Québec, c'est vivre dans une dualité. Contrairement à tant de Québécois de langue anglaise, Scott et les poètes de sa génération n'ont pas feint d'ignorer cette réalité. Scott, A. J. M. Smith et John Glassco sont parmi les premiers à avoir pris au sérieux la culture francophone du Québec et à avoir eu à cœur de la traduire» (Jones 1983: 160).

Dès 1952, Frank Scott avait fait paraître dans Northern Review des versions anglaises des poèmes «La fille maigre» et «En guise de fête» d'Anne Hébert, et avait entrepris de traduire le "Tombeau des rois». Il envoya une première version à l'auteure, qui lui répondit en commentant certains aspects de sa traduction. Cette première version de Scott, suivie du «dialogue» entre la poète et le traducteur et d'une deuxième version de "The Tombs of the Kings", parurent en 1960 dans Les 
écrits du Canada français, précédés d'une note explicative de Jeanne Lapointe, l'initiatrice de ce projet de publication. Quelque temps après, Frank Scott eut la chance de poursuivre le dialogue en tête à tête avec Anne Hébert. D’autres changements furent ainsi apportés à la deuxième version anglaise du «Tombeau des rois ». La troisième version qui en résulta, accompagnée de la correspondance avec Anne Hébert et des deux premières versions, fut publiée à son tour dans The Tamarack Review en 1962. Finalement, toute la correspondance entre les deux poètes et les versions successives de "The Tomb of the Kings» furent réunies dans un livre intitulé Dialogue sur la traduction: à propos $d u$ "Tombeau des rois», avec une préface anglaise de Northrop Frye, traduite en français par Jean Simard.

Le fait que l'échange entre une poète et son traducteur autour d'un travail de traduction poétique soit l'objet d'un livre est un fait unique dans les lettres canadiennes. Évidemment, la notoriété des deux protagonistes n'est pas étrangère à l'affaire. Mais que ce «dialogue» ait commencé dès les années 1950, et ait été mené sur le terrain de la poésie, n'est pas non plus un hasard. Comme l'explique Pierre Popovic, on croit souvent, à tort, que la prose est «l'espace par excellence où l'idéologie sévit et la poésie celui où elle s'effondre». Mais le statut de la poésie au sein du champ littéraire, qui en fait la plus haute expression artistique, «suggère au contraire que, s'il devait être un lieu plutôt qu'un autre où l'idéologie agit à pleine capacité, ce lieu serait sans aucun doute celui du poème» (Popovic 1992: 377-378).

De plus, il semble que la poésie d'Anne Hébert en particulier ait eu pour Scott une signification non négligeable. Bien sûr, en la traduisant, Frank Scott contribuait à faire connaître aux siens un «produit» littéraire du Canada français. Il exprime d'ailleurs le souhait que ses traductions éveillent chez leurs lecteurs le désir de jeter un regard approfondi sur les œuvres originales (Scott 1962: 9). Mais si Scott a voulu traduire Anne Hébert, c'est aussi que son ouvre, et en particulier le «Tombeau des rois», avait une résonance particulière pour lui. Le texte de littérature, nous rappelle Pierre Popovic, n'est pas «en dehors de quoi que ce soit», mais «travaille de manière spécifique à l'intérieur du discours social» (Popovic 1992: 390). Dans son analyse du Tombeau des rois, celui-ci nous montre que l'impuissance notée dans ce recueil d'Anne Hébert «caractérise la situation de l'individu dans le cadre social» (Popovic 1992 : 383) et que se profile, en condensé, dans le poème éponyme, «la critique radicale de tout un système d'éducation» (Popovic 1992: 409).

Popovic montre de façon convaincante que, vu sous l'angle d'une sociologie de l'écriture, ce recueil d'Anne Hébert, qui pouvait sembler au premier abord hors du temps et du monde, dans un espace où «la poésie n'a d'autre loi qu'elle-même» (Popovic 1992: 371), se révèle au contraire traversé de discours sociaux entrelacés. En fait, le Tombeau des rois tamise « un corpus discursif imprécisé dont les contours englobent l'automatisme, Cité libre, La Nouvelle Relève, l'Hexagone, c'est-à-dire toute la frange du dit qui, ouvertement ou indirectement, va à contre-courant du discours de légitimation traditionnelle» (Popovic 1992: 392).

On peut supposer que Frank Scott était sensible à cette remise en question du discours traditionnel inscrite dans l'œuvre hébertienne. Popovic souligne d'ailleurs que ce sont les renseignements fournis par la poète lors de la traduction, par Scott, du «Tombeau des rois» qui ont aidé à mettre en lumière la critique de l'idéologie traditionnelle que contenait ce poème (Popovic 1992: 372). Scott fut sans doute particulièrement réceptif au discours citélibriste qui s'y profilait, car cet «alliage d'un 
humanisme religieux et d'un libéralisme à l'anglaise », selon la formule d'André-J. Bélanger (cité dans Popovic 1992: 76), renfermait des valeurs qui lui étaient chères. Ainsi, traduire, c'est aller à la rencontre de l'autre, mais c'est aussi beaucoup aller à la rencontre de soi-même, se reconnaître dans l'autre.

Quoi qu'il en soit, le «Tombeau des rois» a accompagné Frank Scott pendant une bonne trentaine d'années. C'est d'ailleurs la lecture et la traduction des poèmes d'Anne Hébert qui l'ont conduit vers les autres poètes québécois (Scott 1977: v). Fidèle à l'idée que toute traduction, comme tout poème d'ailleurs, n'est jamais terminée, seulement abandonnée, Scott continua jusqu'en 1984, soit peu de temps avant sa mort, à réviser "The Tomb of the Kings» (Djwa 1987: 376). Ce poème, qui était en quelque sorte devenu le sien, a d'ailleurs été incorporé à ses œuvres complètes. Pourtant, en présentant sa troisième version du «Tombeau des rois » dans The Tamarack Review, en 1962, il avait affirmé que d'autres révisions étaient fort improbables...

Dans un numéro de Meta paru en mars 1969, le poète et traducteur John Glassco signe un article intitulé «The Opaque Medium», consacré à la traduction de la poésie canadienne-française. Glassco note que la poésie d'expression française au Canada connaît la plus importante révolution et renaissance de son histoire. Toutefois, il constate que la traduction poétique est souvent dénigrée. C'est un acte de communication incompris, opaque. On prend plaisir, dit-il, à répéter la remarque de Robert Frost, selon laquelle c'est la poésie elle-même qui se perd dans le procès de traduction. On va jusqu'à se demander si cela vaut la peine de traduire de la poésie. Mais cela ne revient-il pas à se demander pourquoi on se donne la peine d'en écrire?

Saint-Denys Garneau, rappelle Glassco, s'est bien vite rendu compte qu'il se trahissait lui-même en écrivant, qu'il n'arrivait pas à communiquer son expérience. Pourtant, il n'en continuait pas moins à écrire. De la même façon, le traducteur, habitué à être assimilé à un traître, n'en continue pas moins à traduire - au sens religieux ancien de transmission (conveyance) ou de transformation (assumption) la vision du monde qu'il a reçue du poème et à communiquer son expérience aux locuteurs d'une autre langue. Glassco ne traduit donc pas pour faciliter les relations entre les groupes linguistiques, mais pour transmettre une expérience esthétique. Ce qui lui importe, c'est que la vision du monde du poète soit communiquée. Quand son entreprise est un franc succès, écrit Glassco, comme cela arrive parfois, le sentiment d'accomplissement qu'éprouve alors le traducteur est comparable à celui que procure la création poétique. Dans les cas moins heureux, il aura, si l'on peut dire, construit une espèce de pont ( «he has made a bridge of sorts»).

Glassco récapitule ensuite l'histoire de la traduction «sérieuse» de poésie canadienne-française, qu'il fait remonter à un peu plus d'une douzaine d'années. C'est donc une tradition encore très jeune. Les brochures ronéotypées, produites en 1955-1956 à Iroquois Falls par Jean Beaupré et Gael Turnbull, constituent selon lui la première entreprise collective de traduction de poésie.

Glassco donne comme exemple de traductions de piètre qualité celles que propose George Ross Roy, en 1958, dans Douze poètes modernes du Canada français/ Twelve Modern French Canadian Poets, édition bilingue parue à Toronto chez Ryerson. Puis viennent les traductions de poèmes de Nelligan par P. F. Widdows, en 1960, et 
enfin celles de F. R. Scott. Glassco qualifie Scott de premier véritable traducteur de poésie au Canada, d'une part, parce qu'il fut le premier à traduire Saint-Denys Garneau, Anne Hébert, Gilles Hénault, Pierre Trottier, Jean-Guy Pilon et Roland Giguère, d'autre part, parce que ses versions sont exemplaires. Glassco en profite pour annoncer la parution prochaine, chez Oxford University Press, de son anthologie The Poetry of French Canada in Translation (1970), ouvrage qui consacrera son propre rôle de premier plan dans le domaine de la traduction littéraire au Canada.

La dernière partie du texte de Glassco est un plaidoyer en faveur de la traduction poétique de qualité. Le traducteur est constamment tiraillé entre la fidélité au texte original et le devoir de produire un texte véritablement poétique. Aussi, pour Glassco, le traducteur doit être lui-même poète. Après avoir donné quelques exemples de mauvaise traduction, il conclut en disant que les poètes devraient pouvoir empêcher la publication de telles traductions, qui défigurent des œuvres d'une grande qualité et qui leur font du tort. Malheureusement, ces traductions sont souvent publiées à l'insu des poètes et ne font qu'accréditer l'adage italien qui assimile le traducteur à un traître. Il faut faire comprendre aux organismes subventionnaires, écrit Glassco, que la traduction de poésie exige des compétences linguistiques et poétiques: il s'agit de conditions sine qua non ${ }^{5}$.

Dans l'introduction à son anthologie The Poetry of French Canada in Translation, Glassco reprend l'essentiel de son article «The Opaque Medium », paru l'année précédente dans Meta. Il ajoute à ses remarques sur la traduction quelques commentaires sur la poésie canadienne-française elle-même, celle-ci étant selon lui une poésie de l'exil - exil de la France, mais aussi de l'Amérique du Nord.

La position de John Glassco comme traducteur est intéressante à maints égards. Il faut souligner d'emblée le fait qu'il ne s'en tient pas au «simple fait» de traduire. En signant introductions et préfaces, il intervient, entre le texte original et son nouveau public de langue anglaise, pour rappeler notamment à ce dernier que c'est lui qui leur donne à lire ce nouvel objet. Glassco traducteur est bien loin de renoncer à son statut d'éminent homme de lettres. Ne pose-t-il pas lui-même qu'il faut être poète pour traduire de la poésie? Le traducteur n'est assurément pas ici dans une posture de soumission devant son «modèle». Il est, comme lui, créateur, et capable d'exercer à l'endroit des textes qu'il traduit une fonction critique aiguisée. Peut-être peut-on interpréter la moins grande empathie que témoigne Glassco à l'égard des poètes de l'Hexagone et de Parti Pris comme un signe d'agacement devant le refus de l'Autre, c'est-à-dire de lui-même, sous-jacent à leur poésie.

Glassco avoue laisser sentir sa présence dans sa manière même de traduire, ce qui est inévitable, selon lui. Il explique en effet, dans son «Introduction» aux Complete Poems of Saint-Denys Garneau, qu'une coloration personnelle est inéluctable en traduction, puisque celle-ci est faite par des hommes et des femmes, et non pas par des machines: «En traduisant ces poèmes, dit-il, j’ai suivi une ligne de conduite qui ne pouvait manquer d'entraîner l'intrusion de ma propre personnalité. [...] Ces traductions sont fidèles; elles ne sont pas littérales » (Glassco 1975: 17). Il est intéressant de mettre cette affirmation en parallèle avec celle de Frank Scott, qui opte, lui, pour la littéralité.

Glassco explique aussi le choix éditorial qu'il a fait de ne présenter que ses traductions, sans les textes originaux. Selon lui, le poème traduit doit exister pour luimême et être lu avec plaisir par ceux qui ne connaissent pas l'original. On ne peut 
certes accuser Glassco d'oblitérer l'origine des textes qu'il traduit, lui qui parle en long et en large, dans son "Introduction», de Saint-Denys Garneau, d'ailleurs présenté de façon très éloquente. Mais il reste que ce choix s'accorde avec son positionnement général comme traducteur de poésie canadienne-française.

Cet aperçu très sommaire et incomplet du travail remarquable entrepris il y a quelques décennies par Phil Stratford, Frank Scott et John Glassco nous permet de cerner les paramètres fondamentaux de l'activité traduisante. Au point de départ, il y aurait le désir de traduction, senti comme une véritable nécessité. À cet égard, l'histoire de la traduction littéraire au Canada doit beaucoup à Phil Stratford, qui a fait naître chez plus d'un le désir impérieux de se lancer dans cette aventure langagière. Ensuite, viendrait le positionnement du traducteur par rapport à l'œuvre originale: avec Scott et Glassco, nous avons deux beaux exemples de sourcier et de cibliste. Nous voyons ainsi se poursuivre, sur la scène canadienne, le sempiternel débat entre adeptes du verre transparent et fervents du verre coloré.

$\mathrm{Au}$ risque de se mouiller, ces trois traducteurs ont su «traverser le torrent sur les roches », ont osé «quitter cette chose pour celle-là» et, avec le poète Saint-Denys Garneau, ils ont pu dire, en de rares moments de grâce: "Je trouve l'équilibre impondérable entre les deux / C’est là sans appui que je me repose».

\section{NOTES}

1. Originaire de l'Ontario, Philip Stratford a fait ses études à la University of Western Ontario et à la Sorbonne. Il devient professeur de littérature canadienne à l'Université de Montréal en 1964. Il est membre fondateur de l'Association des traducteurs littéraires du Canada en 1975 et reçoit en 1988 le prix de traduction du Gouverneur Général.

2. Titre retraduit par Jean Simard (Cercle du livre de France, 1976). En fait, comme il le note dans sa Bibliographie parue en 1975, un autre roman de Richler, A Choice of Enemies (1957), avait été traduit Le choix des ennemis (1959).

3. Nous avons traduit toutes les citations en langue originale du présent article.

4. Cette position diffère de celle qu'adoptera John Glassco au moment de préparer son anthologie de poésie canadienne-française en traduction, The Poetry of French Canada in Translation: dans son introduction, Glassco explique en effet que des poèmes importants, mais rebelles à la traduction, ont été omis parce qu'on n'a pas réussi à en produire de version anglaise satisfaisante. Par contre, ont été incluses de belles traductions de poèmes moins représentatifs. En un sens, écrit Glassco, il s'agit plus d'une anthologie de traductions poétiques que d'une anthologie de poèmes en traduction (xxiv).

5. Glassco n'est pas le seul traducteur à s'être élevé contre la mauvaise qualité de certaines traductions. En 1967, Patricia Claxton, qui sera plus tard membre fondatrice de l'Association des traducteurs littéraires (en 1975) et traductrice de Gabrielle Roy, signe dans Meta (12-1, mars 1967, p. 9-13) un article intitulé "Culture Vulture», dans lequel elle déplore la qualité pitoyable de certaines traductions. Elle puise surtout ses exemples dans la version anglaise de Prochain épisode, par Penny Gail Williams (McClelland \& Stewart, 1967), dont des extraits furent d'abord publiés dans Maclean's Magazine. Selon Madame Claxton, le grand défaut de cette traduction est d'être calquée sur le français, tant du point de vue de la syntaxe que de la ponctuation. Le Conseil des arts, dit-elle, ne doit pas subventionner la médiocrité. Chacune des deux cultures ne prendra l'habitude de jeter un coup d'œil de l'autre côté de la clôture que si elle y trouve son plaisir. Madame Claxton propose enfin la création d'un prix annuel de traduction littéraire qui ne serait pas attribué les années où aucune traduction remarquable n'a été produite. (Les prix de traduction du Conseil des Arts ont été créés en 1974.) 


\section{RÉFÉRENCES}

Bergeron, Léandre (1963): «Une heureuse initiative», Canadian Literature, 18, automne 1963, p. 75-76.

Djwa, Sandra (1987): The Politics of the Imagination, Toronto, McClelland \& Stewart.

Glassco, John (1969): «The Opaque Medium», Meta, 14-1, mars 1969, p. 27-30.

—, dir. (1970): The Poetry of French Canada in Translation, Oxford, Oxford University Press.

- (1975): Complete Poems of Saint-Denys Garneau, Ottawa, Oberon Press.

Grandpré, Chantal de (1985): «La canadianisation de la littérature québécoise: le cas Aquin», Liberté 159, 27-3, juin 1985, p. 50-59.

Jones, D. G. (1983) : «F. R. Scott as Translator», On F. R. Scott. Essays on His Contributions to Law, Literature, and Politics (Sandra Djwa et R. St J. Macdonald, dir.), Kingston et Montréal, McGill-Queen's University Press, p. 160-164.

Popovic, Pierre (1992): La contradiction du poème, Candiac, Éditions Balzac.

Scotт, F. R. (1962) : St-Denys Garneau/Anne Hébert. Translations/Traductions, Vancouver, Klanak Press.

—, trad. (1977): Poems of French Canada, Burnaby, Blackfish Press.

— et Anne Hébert (1970), Dialogue sur la traduction, Montréal, HMH.

Stratford, Phil (1968): «French-Canadian Literature in Translation», Meta, 13-4, décembre 1968, p. 180-187.

- (1977): Bibliography of Canadian Books in Translation: French to English and English to French / Bibliographie de livres canadiens traduits de l'anglais au français et du français à l'anglais, Ottawa, HRCC/CCRH. 